\title{
Accumulation of biologically active phenolic acids in agitated shoot cultures of three Hypericum perforatum cultivars: 'Elixir', 'Helos' and 'Topas'
}

\author{
Inga Kwiecień $^{1}$ - Aleksandra Szydłowska ${ }^{1} \cdot$ Beata Kawka $^{1}$ • \\ Ludger Beerhues ${ }^{2} \cdot$ Halina Ekiert ${ }^{1}$
}

Received: 21 March 2015/Accepted: 22 July 2015/Published online: 31 July 2015

(c) The Author(s) 2015. This article is published with open access at Springerlink.com

\begin{abstract}
Agitated shoot cultures of three Hypericum perforatum cultivars: 'Elixir', 'Helos' and 'Topas' were established and maintained on Linsmaier and Skoog (LS) and Murashige and Skoog (MS) media containing varying concentrations $\left(0.1-3.0 \mathrm{mg} \mathrm{l}^{-1}\right)$ of $\alpha$-naphthaleneacetic acid (NAA) and 6-benzyladenine (BA). In methanolic extracts of the biomass, the amounts of free phenolic acids and cinnamic acid were determined by HPLC. Six of the seventeen compounds analyzed were detected in all the extracts: 3,4-dihydroxyphenylacetic acid, $p$-coumaric acid, protocatechuic acid, syringic acid, and two depsideschlorogenic and neochlorogenic acids. The amounts of individual compounds and the total amount of phenolic acids depended on the media variants used and the increases ranged from 1.2- to 15.0-fold and from 1.2- to 1.9-fold, respectively. The maximum total amounts in the biomass of 'Elixir', 'Helos' and 'Topas' were 212, 222, and $191 \mathrm{mg}$ $100 \mathrm{~g}^{-1} \mathrm{DW}$, respectively. The main metabolites in all the cultivars were neochlorogenic acid (max. $118.81 \mathrm{mg}$
\end{abstract}

Dedicated to the memory of Prof. dr. hab. Jan Krzek, Head of Chair and Department of Inorganic Chemistry, Jagiellonian University, Collegium Medicum, Dean of Pharmaceutical Faculty 2008-2015, who died on 1 February 2015.

Inga Kwiecień

inga.kwiecien@uj.edu.pl

Halina Ekiert

mfekiert@cyf-kr.edu.pl

1 Chair and Department of Pharmaceutical Botany, Jagiellonian University, Collegium Medicum, Medyczna Street 9, 30-688 Kraków, Poland

2 Institut für Pharmazeutische Biologie, Technische Universität Braunschweig, Mendelssohnstrasse 1, 38106 Braunschweig, Germany
$100 \mathrm{~g}^{-1} \mathrm{DW}$ ) and 3,4-dihydroxyphenylacetic acid (max. $\left.129.29 \mathrm{mg} 100 \mathrm{~g}^{-1} \mathrm{DW}\right)$. Three metabolites that also accumulated in high amounts were protocatechuic acid (max. $21.43 \mathrm{mg} 100 \mathrm{~g}^{-1} \mathrm{DW}$ ), syringic acid (max. $23.63 \mathrm{mg}$ $100 \mathrm{~g}^{-1} \mathrm{DW}$ ) and chlorogenic acid (max. $18.79 \mathrm{mg} 100 \mathrm{~g}^{-1}$ DW). The richest source of the estimated compounds and hence a potential biotechnological platform were the shoots of 'Helos' cultivated on LS and MS media containing low concentrations of NAA and BA (0.1-1.0 $\left.\mathrm{mg} \mathrm{l}^{-1}\right)$.

Keywords 3,4-Dihydroxyphenylacetic acid · HPLC analysis · In vitro cultures · Neochlorogenic acid . Secondary metabolites $\cdot$ St. John's Wort

\begin{tabular}{|c|c|}
\hline \multicolumn{2}{|c|}{ Abbreviations } \\
\hline BA & 6-Benzyladenine \\
\hline DW & Dry weight \\
\hline HPLC & High-performance liquid chromatography \\
\hline LS & Linsmaier and Skoog \\
\hline MS & Murashige and Skoog \\
\hline NAA & $\alpha$-Naphthaleneacetic acid \\
\hline
\end{tabular}

\section{Introduction}

Hypericum perforatum L. (St. John's Wort) is one of the best known medicinal plant species used in both traditional and modern medicine. It is characterized by a rich chemical composition, and the bioactive compounds of the herb (Herba Hyperici) represent biogenetically different groups of plant secondary metabolites. The main constituents are derivatives of anthraquinones (hypericins), flavonoids, tannins, phloroglucinols (hyperforins), xanthones, terpenes (essential oil), and phenolic acids. The phenolic acids are 
represented by caffeic, $p$-coumaric, ferulic, $p$-hydroxybenzoic and vanillic acids as well as the depsidechlorogenic acid (Barnes et al. 2007).

Plants originating from natural habitats in different regions of Europe have been found to contain mostly chlorogenic (Cirak et al. 2007) and neochlorogenic acids (Silva et al. 2008), caffeic and protocatechuic acids (Dias et al. 1999; Tatsis et al. 2007), and isomers of $p$-coumaroylquinic acid (Jurgenliemk and Nahrstedt 2002). By comparison, preliminary studies conducted at our department in Kraków have revealed that, in addition to caffeic, chlorogenic, $p$-hydroxybenzoic, protocatechuic and vanillic acids, syringic acid is also present in the herbal raw material supplied by two Polish herbal companies.

Phenolic acids have valuable biological properties, including antioxidant, immunostimulant, anticancer, antiaggregatory and anti-inflammatory activities. In particular, the antioxidant properties of phenolic acids attract an enormous amount of interest from both the pharmaceutical and cosmetics industries (Khadem and Marles 2010).

To date, the quite extensive biotechnological research on $H$. perforatum, and other species of the genus Hypericum, has primarily focused on studying the biosynthesis and accumulation of hypericins, hyperforins, xanthones and flavonoids (Beerhues 2011; Charchoglyan et al. 2007; Don Palmer and Keller 2011; Karting et al. 1996; Liu et al. 2007). In addition, numerous micropropagation protocols have been developed (Goel et al. 2009; Savio et al. 2012). Some of them can be useful in the production of elite varieties with predictable phytochemical profiles for pharmaceutical purposes (Murch and Saxena 2006). The biosynthesis and accumulation of phenolic acids in in vitro cultures of $H$. perforatum have so far been the subject of isolated biotechnological studies. In adventitious root biomass cultivated in a balloon-type airlift bioreactor, high amounts of chlorogenic acid $\left(0.80 \mathrm{mg} \mathrm{g}^{-1}\right)$ and, among flavonoids, of quercetin and hyperoside have been reported (Cui et al. 2010). Other investigations have revealed the accumulation of neochlorogenic and ferulic acids (Dias et al. 1999; Pasqua et al. 2003) in the biomass of $H$. perforatum cultured in vitro.

Preliminary studies of the accumulation of free phenolic acids in agar cultures of $H$. perforatum were conducted earlier at our department in Kraków, and similar investigations have recently been performed with agar cultures of three cultivars-'Elixir', 'Helos' and 'Topas'. The preliminary results were sufficiently encouraging to continue and conduct these studies within a wider scope. An additional incentive to undertake further research were the biotechnological achievements concerning the accumulation of this particular group of plant secondary metabolites, especially of rosmarinic acid (Makri and Kintzios 2004; Ekiert et al. 2013).

The aim of the present study was to establish agitated shoot cultures of three $H$. perforatum cultivars ('Elixir',
'Helos', 'Topas') and to optimize the conditions for the growth of biomass and accumulation of free phenolic acids. A precursor for one subgroup of these compounds, cinnamic acid, was included. Altogether, seventeen compounds were analyzed by HPLC. The study aimed at nominating the best 'growth' and 'production' media for the three cultivars. The cultures were performed on four variants each of Linsmaier and Skoog (LS) medium and Murashige and Skoog (MS) medium containing varying concentrations (0.1-3.0 $\left.\mathrm{mg} \mathrm{l}^{-1}\right)$ of plant growth regulators, namely the auxin-NAA and the cytokinin-BA. In order to assess the biosynthetic potential of the tested cultivars, the cultures were additionally analyzed for hypericin content. Our previous studies of the accumulation of hypericin with densitometric detection and of indole compounds, such as serotonin, melatonin, indole-3-acetic acid, L-tryptophan and 5-hydroxy-L-tryptophan, in agar shoot cultures of the three $H$. perforatum cultivars had indicated a remarkable biosynthetic potential, which, however, differed significantly between the cultivars (Muszyńska et al. 2014).

\section{Materials and methods}

\section{Plant material}

The in vitro shoot cultures of the three Hypericum perforatum L. cultivars: 'Elixir', 'Helos', and 'Topas' were established in 2007 at the Institut für Pharmazeutische Biologie, Technische Universität Braunschweig (Germany). Seeds had been purchased from Richters Herbs (GooDMood, Canada). They were surface-sterilized with $70 \%$ ethanol for 2 min and $6 \%$ sodium hypochlorite for $5 \mathrm{~min}$. After being rinsed three times with sterile water (for 10 min each time), the seeds were placed on a hormone-free Murashige and Skoog (MS) medium (Murashige and Skoog 1962) at $25^{\circ} \mathrm{C}$. Seeds germination started within 3 weeks under a $16 / 8 \mathrm{~h}$ light/dark period $\left(16 \mu \mathrm{mol} \mathrm{m} \mathrm{m}^{-2} \mathrm{~s}^{-1}\right)$.

The resulting shoot cultures of 'Elixir', 'Helos', and 'Topas' were maintained on solid MS medium supplemented with $0.5 \mathrm{mg} \mathrm{l}^{-1} \mathrm{NAA}$ and $0.5 \mathrm{mg}^{-1} \mathrm{BA}$ (pH 5.7). The cultures were grown under constant artificial light $\left(16 \mu \mathrm{mol} \mathrm{m}{ }^{-2} \mathrm{~s}^{-1}\right.$, LF-40 W lamp, daylight, Piła) at $25 \pm 2{ }^{\circ} \mathrm{C}$. Shoots were subcultured every 6 weeks (Fig. 1a, b).

\section{Experimental cultures}

Agitated shoot cultures of the three $H$. perforatum cultivars: 'Elixir', 'Helos' and 'Topas' were performed on two types of medium: MS and Linsmaier and Skoog (LS) medium (Linsmaier and Skoog 1965), which contained different amounts of the plant growth regulators NAA and BA $\left(\mathrm{mg}^{-1}\right)$ : 0.1 and $0.1,1.0$ and 1.0, 2.0 and 2.0, and 3.0 
Fig. 1 In vitro cultures of Hypericum perforatum 'Helos' cultivar. a, b Initial agar shoot culture (MS, $0.5 \mathrm{mg}^{-1} \mathrm{NAA}$ and $\left.0.5 \mathrm{mg} \mathrm{l}^{-1} \mathrm{BA}\right), \mathbf{c}$, d experimental shoot agitated culture (LS, $0.1 \mathrm{mg}^{-1} \mathrm{NAA}$ and $0.1 \mathrm{mg} \mathrm{l}^{-1} \mathrm{BA}$ )
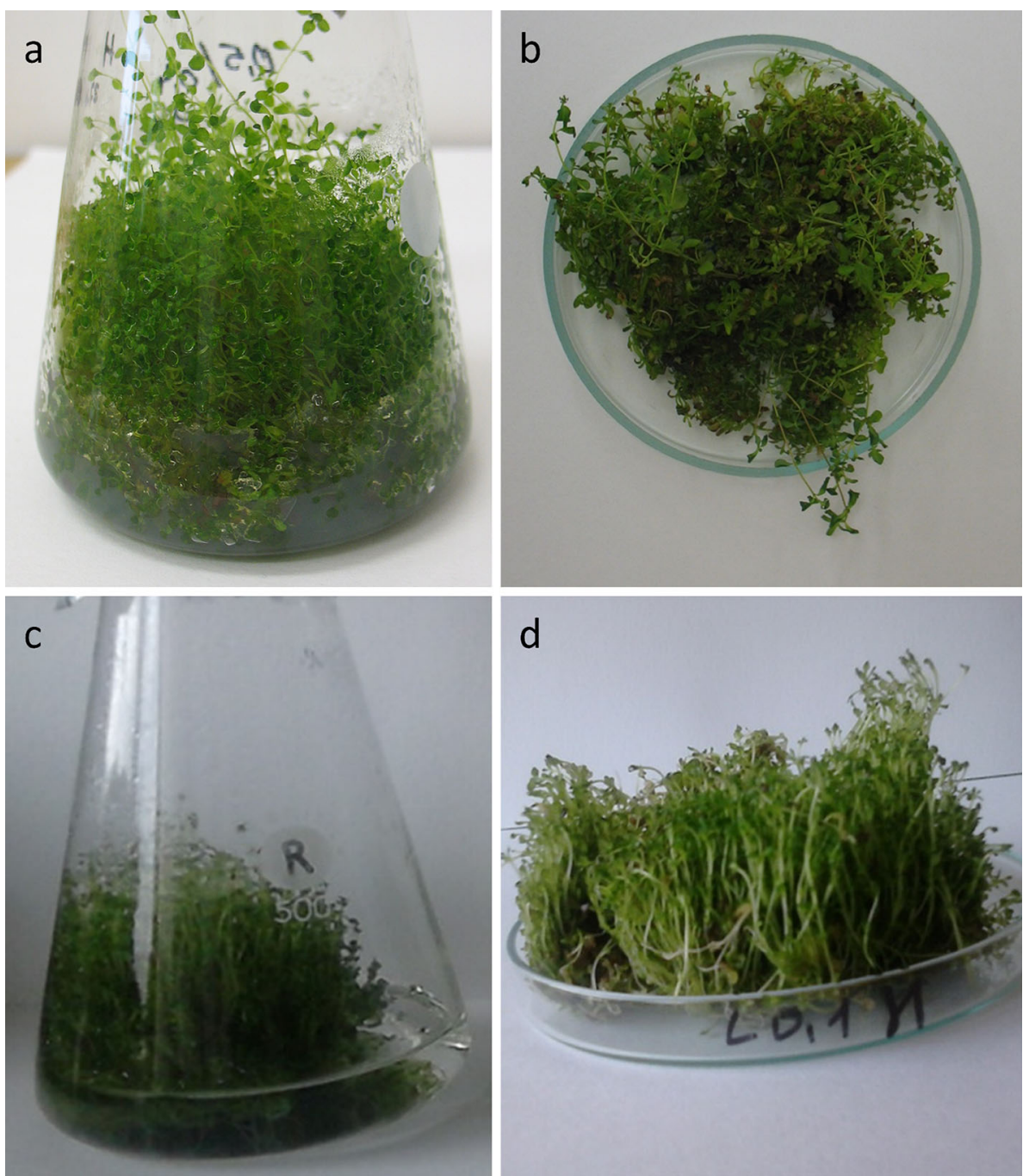

and 3.0, respectively. Erlenmeyer flasks $(500 \mathrm{ml})$ were filled with $150 \mathrm{ml}$ of medium. The agitated cultures were initiated with 6-week-old shoot clusters. The inoculum for the cultures was $1 \mathrm{~g}$ of fresh biomass per flask. Three subculture cycles (3 weeks each) were carried out before the main experiment. The cultures (three series) were maintained under the same light and temperature conditions as the initial cultures, on an Altel rotary shaker operating at $140 \mathrm{rpm}$ with a vibration amplitude of $35 \mathrm{~mm}$ for 3 weeks (Fig. 1c, d).

\section{Extraction and HPLC analysis}

The biomass was dried in fresh air at $25 \pm 2{ }^{\circ} \mathrm{C}$, ground, and extracted with boiling methanol $(50 \mathrm{ml})$ for $3 \mathrm{~h}$. The methanol was evaporated and the residue was dissolved in HPLC-grade methanol. The methanolic extracts were analyzed using modified HPLC method (Ellnain-Wojtaszek and
Zgórka 1999). An HPLC-system (Merck-Hitachi) and a Purospher RP-18e analytical column $(4 \times 250 \mathrm{~mm}, 5 \mu \mathrm{m}$; Merck) were used. The mobile phase consisted of methanol (A) and $0.5 \%$ acetic acid (B) (gradient elution). The flow rate was $1 \mathrm{ml} \mathrm{min}{ }^{-1}$. Compounds were estimated using a DAD detector. Qualification and quantification analyses were based on comparison with reference substances of caffeic, cinnamic, chlorogenic, $o$-coumaric, $p$-coumaric, 3,4dihydoxyphenylacetic, ferulic, gallic, gentisic, $p$-hydroxybenzoic, neochlorogenic, protocatechuic, rosmarinic, salicylic, sinapic, syringic and vanillic acids (Sigma-Aldrich). Additionally, an analysis of hypericin content was performed using the Sigma-Aldrich standard.

\section{Statistical analysis}

The obtained results are expressed as the mean $\pm \mathrm{SD}$ of three independent experiments and determinations. 


\section{Results}

\section{Accumulation of metabolites on the tested variants of LS medium}

The biomass from the agitated shoot cultures of $H$. perforatum ('Elixir', 'Helos' and 'Topas'), which were grown on variants of LS medium containing different concentrations of plant growth regulators, was characterized by a varying morphological appearance. The cultures of all three cultivars on the medium with the lowest level of plant growth regulators $\left(0.1 \mathrm{mg} \mathrm{l}^{-1} \mathrm{NAA}\right.$ and $\left.0.1 \mathrm{mg}^{-1} \mathrm{BA}\right)$ produced numerous green leafy shoots. With an increase in the concentration of plant growth regulators, the biomass became more compact, the shoots were shorter and formed callus tissue at the base. During the 3-week-culture cycles on the tested variants of LS medium, different increases in dry biomass were obtained, which ranged from 7.2 to 8.5 for 'Elixir', from 8.5 to 9.7 for 'Helos', and from 7.8 to 9.1 for 'Topas'. The largest increases were obtained for the cultivar 'Helos' grown on LS media containing $0.1 \mathrm{mg}^{-1}$ NAA and $0.1 \mathrm{mg}^{-1} \mathrm{BA}$ (9.5-fold) and $2 \mathrm{mg}^{-1}$ NAA and $2 \mathrm{mg} \mathrm{l}^{-1}$ BA (9.7-fold)(Fig. 1c, d).

The chromatographic analysis of the extracts from the in vitro biomass of the three cultivars proved the presence of the same seven compounds out of the seventeen that were tested-chlorogenic, $p$-coumaric, 3,4-dihydroxyphenylacetic, neochlorogenic, protocatechuic, syringic and vanillic acids (Fig. 2; Table 1). None of the extracts was found to contain the following acids: caffeic, cinnamic, $o$-coumaric, ferulic, gallic, gentisic, p-hydroxybenzoic, rosmarinic, salicylic and sinapic acids.

The amounts of the individual compounds as well as their total amount in the extracts from the biomass of all three cultivars was clearly dependent on the concentrations of the plant growth regulators in the tested LS medium variants. The total phenolic acids content in the shoots increased, depending on the LS variant, 1.37-fold for 'Elixir', 1.79-fold for 'Helos', and 1.22-fold for 'Topas' (Fig. 3). The maximum total phenolic acids content for 'Elixir' (161.6 mg $100 \mathrm{~g}^{-1} \mathrm{DW}$ ) was found on LS medium containing auxin and cytokinin at $0.1 \mathrm{mg} \mathrm{l}^{-1}$ each. An almost identical maximum total amount of metabolites $\left(160.5 \mathrm{mg} 100 \mathrm{~g}^{-1} \mathrm{DW}\right)$ was found in the shoots of 'Topas' growing on LS medium containing $1 \mathrm{mg}^{-1}$ NAA and $1 \mathrm{mg} \mathrm{l}^{-1} \mathrm{BA}$. The highest maximum total amount of the compounds analyzed (222.4 mg $\left.100 \mathrm{~g}^{-1} \mathrm{DW}\right)$ was present in extracts from the shoots of 'Helos', which also grew on LS medium containing NAA and BA at $1.0 \mathrm{mg}^{-1}$ each (Fig. 3).

The largest amounts accumulating in the biomass of all three cultivars were those of 3,4-dihydroxyphenylacetic acid
(31.66-29.29 mg $\left.100 \mathrm{~g}^{-1} \mathrm{DW}\right)$ and neochlorogenic acid (24.22-87.77 mg $\left.100 \mathrm{~g}^{-1} \mathrm{DW}\right)$. Acids that accumulated in lower amounts were chlorogenic $\left(5.35-15.34 \mathrm{mg}_{100 \mathrm{~g}^{-1}}\right.$ DW), protocatechuic (16.60-21.43 mg $\left.100 \mathrm{~g}^{-1} \mathrm{DW}\right)$ and syringic (10.23-20.37 mg $\left.100 \mathrm{~g}^{-1} \mathrm{DW}\right)$. Even lower were the amounts of $p$-coumaric acid (3.51-12.56 mg $100 \mathrm{~g}^{-1}$ DW). Vanillic acid was present in very small amounts (0.01-1.56 mg $\left.100 \mathrm{~g}^{-1} \mathrm{DW}\right)$ and was not detectable in two extracts (Table 1$)$.

The amount of hypericin in the biomass of H. perforatum in vitro cultures grown on variants of LS medium ranged from 3.06 to $10.98 \mathrm{mg} 100 \mathrm{~g}^{-1} \mathrm{DW}$. The highest amounts were present in extracts from the shoots of 'Elixir'. The lowest amount of this metabolite was in 'Helos' (max. 5.88 mg $100 \mathrm{~g}^{-1} \mathrm{DW}$ ) (Table 1).

\section{Accumulation of metabolites on the tested variants of MS medium}

Like on the tested variants of LS medium, the biomass from the agitated cultures of 'Elixir', 'Topas' and 'Helos' was also morphologically diverse on the tested variants of MS medium. On the medium containing auxin and cytokinin at $0.1 \mathrm{mg} \mathrm{l}^{-1}$ each, the biomass consisted of numerous green leafy shoots. An increase in the concentrations of the plant growth regulators in the medium resulted in morphological changes. The shoots became shorter, more compact, with callus tissue forming at their base. The increases in dry biomass during 3-week-growth cycles on the tested variants of MS medium also varied, namely from 6.4 to 8.6 for 'Elixir', from 8.5 to 9.2 for 'Helos', and from 5.9 to 8.2 for 'Topas'.

As in the case of the LS variants, the highest increases were obtained for the cultivar 'Helos'. They were particularly pronounced (9.2- and 8.8-fold) on two variants of MS medium containing auxin and cytokinin $0.1 \mathrm{mg} \mathrm{l}^{-1}$ and $1 \mathrm{mg} \mathrm{l}^{-1}$ each. A high increase (8.6-fold) was also recorded for the biomass of 'Elixir' on MS medium containing $1.0 \mathrm{mg} \mathrm{l}^{-1} \mathrm{NAA}$ and $1.0 \mathrm{mg} \mathrm{l}^{-1} \mathrm{BA}$.

The analyzed extracts from the biomass growing on the tested variants of MS medium had the same qualitative composition of phenolic acids as in the case of the variants of LS medium (Table 2). Of the seventeen compounds analyzed, the presence of seven was confirmed: chlorogenic, $p$-coumaric, 3,4-dihydroxyphenylacetic, neochlorogenic, protocatechuic, syringic, and vanillic acids. The amounts of the individual phenolic acids as well as their total amount in the analyzed extracts from the biomass of the cultivars studied varied greatly, depending on the MS medium variant (Table 2).

The total amount of metabolites increased for the biomass of individual cultivars as follows: 1.4-fold for 


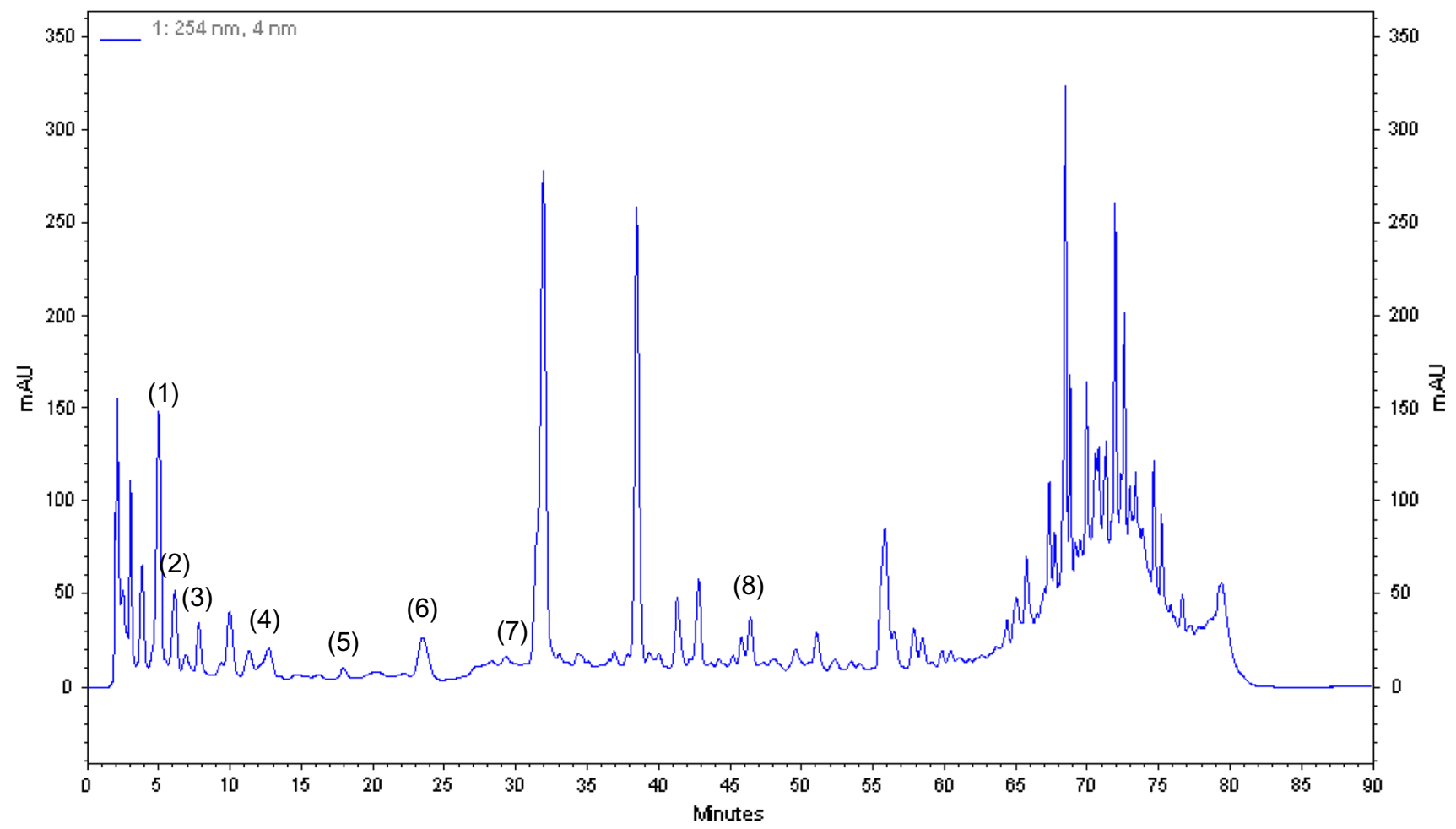

Fig. 2 Chromatogram of methanolic extract of Hypericum perforatum 'Helos'cultivar grown on LS medium $-1.0 \mathrm{mg}^{-1} \mathrm{NAA}$ and $1.0 \mathrm{mg} \mathrm{l}^{-1} \mathrm{BA}$; (1) protocatechuic acid, (2) neochlorogenic acid, (3) 3,4-dihydoxyphenylacetic acid, (4) chlorogenic acid, (5) vanillic acid, (6) syryngic acid, (7) p-coumaric acid, (8) hypericin

cultivar 'Elixir' was the richest source of this metabolite. The highest hypericin content was confirmed in the biomass of the shoots of 'Elixir' growing on MS medium containing $2.0 \mathrm{mg} \mathrm{l}^{-1} \mathrm{NAA}$ and $2.0 \mathrm{mg} \mathrm{l}^{-1} \mathrm{BA}$. In 'Helos' shoot extracts the maximum amount of hypericin was $7.53 \mathrm{mg} 100 \mathrm{~g}^{-1} \mathrm{DW}$ (Table 2).

\section{Discussion}

The biomass of shoots of all three $H$. perforatum cultivars grown in agitated cultures increased considerably. A particularly rapid increase in shoot dry biomass, more than ninefold, during 3-week-growth cycles on the three tested media variants was observed for 'Helos'.

The analysis of extracts from the shoots of the three cultivars studied revealed a qualitatively different composition of phenolic acids in comparison with open-air grown H. perforatum of different origin (Dias et al. 1999; Jurgenliemk and Nahrstedt 2002; Gorzkiewicz and Ekiert 2004, unpublished; Cirak et al. 2007; Tatsis et al. 2007; Silva et al. 2008). The biomass of all the cultivars grown in vitro was notable for the presence of high amounts of 3,4-dihydroxyphenylacetic acid (Tables 1,2).

The concentrations of the tested plant growth regulators, NAA and BA, in culture medium affected considerably the $14.45 \mathrm{mg} 100 \mathrm{~g}^{-1} \mathrm{DW}$. Also on LS medium variants, the 


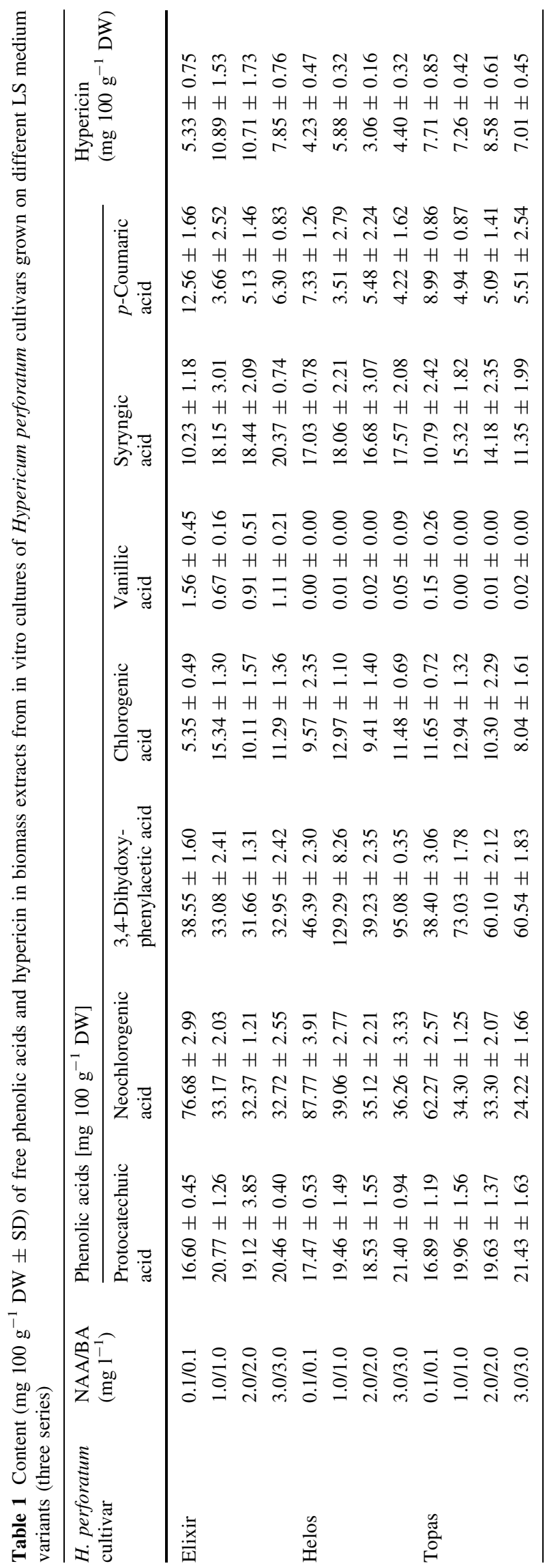

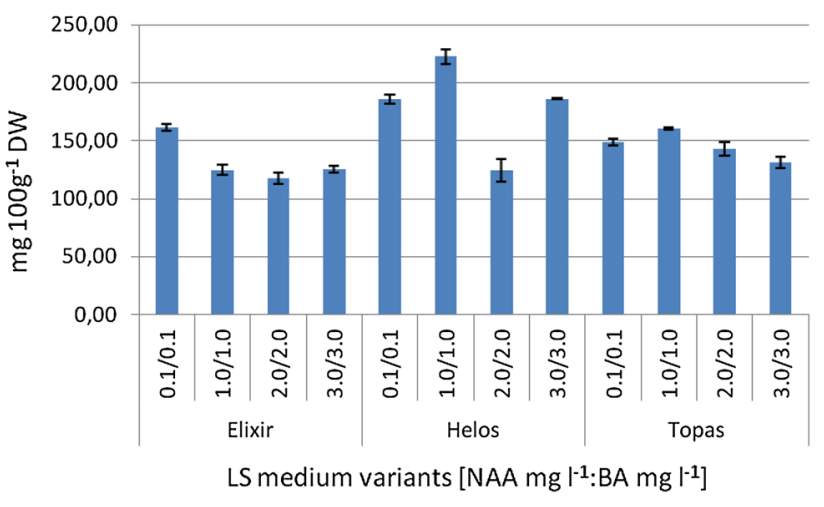

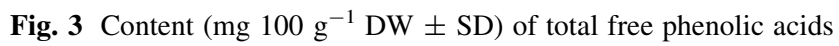
in biomass extracts from in vitro cultures of Hypericum perforatum cultivars grown on LS medium variants (three series)

amounts of individual compounds and consequently their total amount in the cultured biomass. On the tested variants of LS medium, the amounts of individual compounds in the shoots increased from 1.08 to 15.0-fold (Table 1). The total amount of the compounds increased 1.37-, 1.79- and 1.22-fold for 'Elixir', 'Helos' and 'Topas', respectively (Fig. 3). On the tested variants of MS medium, the amounts of individual metabolites increased from 1.14 to 5.19-fold (Table 2). The differences in the total amount of the compounds were also considerable: 1.95-, 1.40- and 1.77-fold, respectively (Fig. 4).

The impact of the concentrations of plant growth regulators in culture media on the accumulation of metabolites is a well-known phenomenon. Our previous studies had demonstrated a clear influence of the concentrations of plant growth regulators on the accumulation of biogenetically different groups of metabolites-coumarins (Ekiert 2004), lignans (Szopa and Ekiert 2011, 2013, 2015), and phenolic acids (Ekiert et al. 2009, 2014; Szopa and Ekiert 2012, 2014; Szopa et al. 2013).

Our studies of in vitro cultures of Aronia melanocarpa have recently also demonstrated the importance of the basic composition of the culture media (LS and MS) on the accumulation of free phenolic acids. Variants of MS medium with the same composition of plant growth regulators as in variants of LS medium allowed us to obtain from 1.61to 2.00-fold higher amounts of free phenolic acids (Szopa et al. 2013; Szopa and Ekiert 2014). In the present study with the three cultivars, the results were different. Only in four out of twelve extracts from the shoots grown on MS variants, the total amount of the compounds was from 1.19 to 1.47 times higher than in the extracts from the shoots grown on identical variants of LS medium.

The amounts of individual compounds as well as their total amounts obtained by us are significant due to the high degree of differentiation of the in vitro cultured biomass. Cultures with a high degree of differentiation usually 


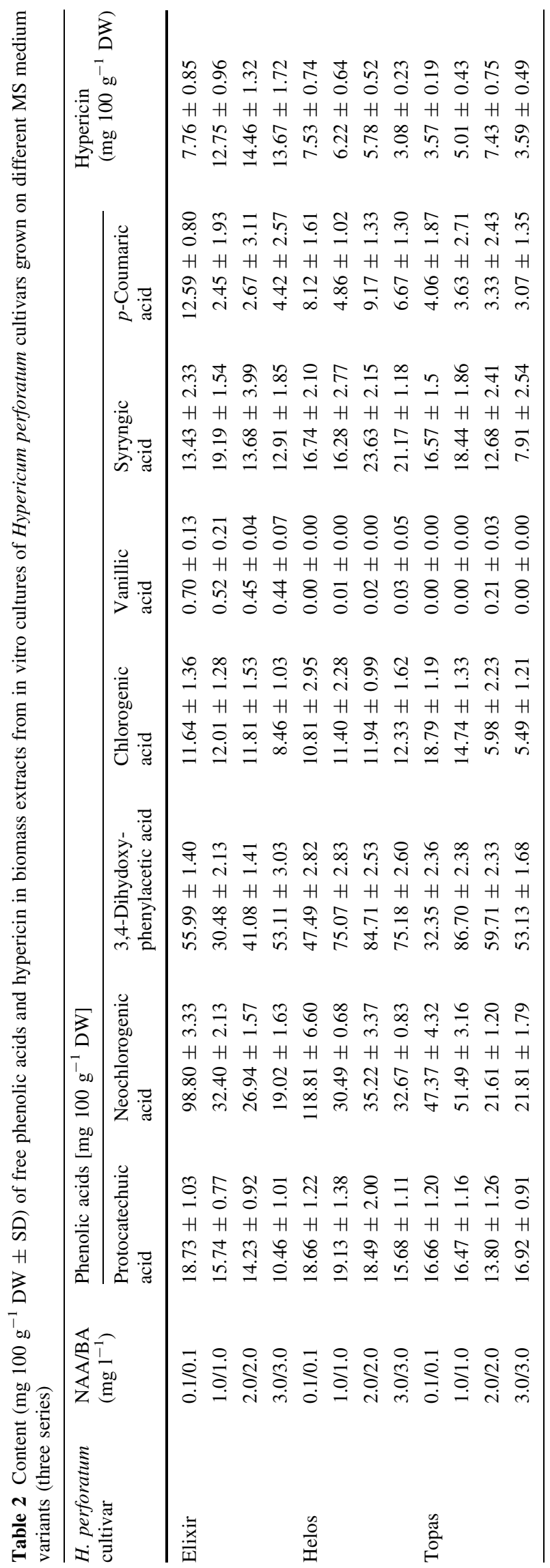

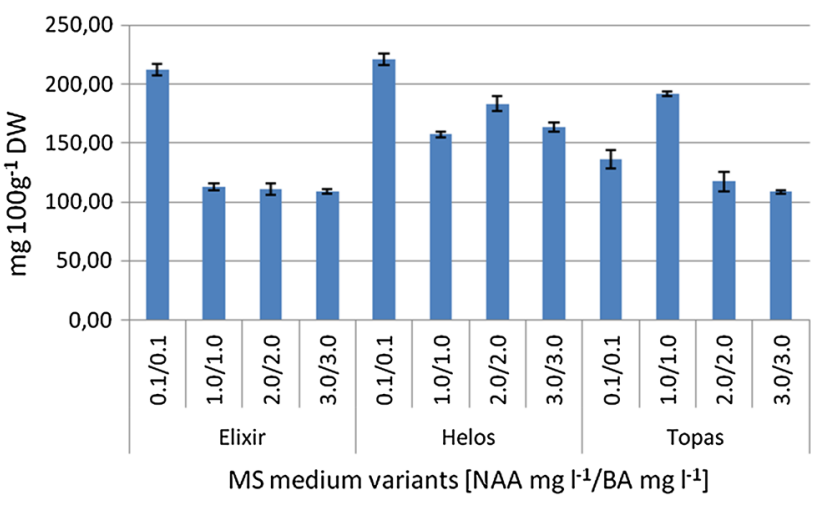

Fig. 4 Content $\left(\mathrm{mg} 100 \mathrm{~g}^{-1} \mathrm{DW} \pm \mathrm{SD}\right.$ ) of total free phenolic acids in biomass extracts from in vitro cultures of Hypericum perforatum cultivars grown on MS medium variants (three series)

provide significantly higher amounts of secondary metabolites than undifferentiated callus cultures. Our previous studies on the accumulation of free phenolic acids have shown significant amounts of these compounds in shoot cultures of Ruta graveolens (Ekiert et al. 2009), shoot-differentiating callus cultures of Ruta graveolens ssp. divaricata (Ekiert et al. 2014), and shoot cultures of Aronia melanocarpa (Szopa et al. 2013; Szopa and Ekiert 2014). Furthermore, our studies have demonstrated the importance of the type of culture on the accumulation of phenolic acids. In our preliminary studies, agar cultures of the three cultivars of $H$. perforatum grown on MS medium variants accumulated several times lower amounts of free phenolic acids (unpublished). These results confirmed the observations of other research teams, which demonstrated that liquid media are better for the accumulation of phenolic compounds in $H$. perforatum in vitro systems (Savio et al. 2012).

Based on the results of the present study, agitated shoot cultures of the cultivar 'Helos' can be nominated as a rich, potential biotechnological source of the bioactive phenolic acids analyzed. As a good 'production' medium, we can propose the variant of LS medium containing $1.0 \mathrm{mg} \mathrm{l}^{-1}$ NAA and $1.0 \mathrm{mg}^{-1} \mathrm{BA}$, and/or the variant of MS medium containing $0.1 \mathrm{mg} \mathrm{l}^{-1} \mathrm{NAA}$ and $0.1 \mathrm{mg} \mathrm{l}^{-1} \mathrm{BA}$. The total amount of the compounds of interest on these media was approx. $220 \mathrm{mg} 100 \mathrm{~g}^{-1}$ DW (Figs. 3, 4). Both nominated media are also good 'growth' media, which guarantee about 9-fold increases in the dry weight of shoots during 3-week-culture cycles. Of the three cultivars studied, the cultivar 'Helos' appears to have the highest enzymatic potential for the biosynthesis of phenolic acids. The shoots of this cultivar grown in vitro accumulate significant amounts of 3,4-dihydroxyphenylacetic acid and neochlorogenic acid (max. $129.29 \mathrm{mg} 100 \mathrm{~g}^{-1} \mathrm{DW}$ and $118.81 \mathrm{mg} 100 \mathrm{~g}^{-1} \mathrm{DW}$, respectively), and also large quantities of other phenolic acids, such as chlorogenic, 
protocatechuic, and syringic (max. 12.97, 21.40, and $23.63 \mathrm{mg} 100 \mathrm{~g}^{-1} \mathrm{DW}$, respectively) (Tables 1, 2). Protocatechuic acid and chlorogenic acid stand out because of their exceptional biological activities. Their antioxidant, antiradical, antibacterial and anticancer properties are proven (Rice-Evans et al. 1996; Sánchez-Maldonado et al. 2011). Moreover, antiproliferative and apoptotic effects of 3,4-dihydroxyphenylacetic acid have been demonstrated (Kampa et al. 2004).

As shown by the qualitative and quantitative analyses of hypericin (performed for comparative purposes), 'Helos' was characterized by a moderate capacity for the biosynthesis of this compound. The highest hypericin content was found in extracts from the shoots of 'Elixir'. Our earlier studies on the accumulation of nonhallucinogenic indole compounds and hypericin in agar cultures of the same three cultivars of $H$. perforatum had also indicated the shoots of 'Helos' to accumulate the highest amounts of indole compounds (serotonin and 5-hydroxy-L-tryptophan) and 'Elixir' as the richest source of hypericin (Muszyńska et al. 2014). The results of ongoing studies confirm the different enzymatic potential of cells from in vitro cultures of the cultivars being analyzed and consequently a different phytochemical profile of the cultured biomass. Our present study has confirmed the substantial amounts of 3,4-dihydroxyphenylacetic acid in the biomass grown in vitro. This is the first report of the presence of this compound in plant in vitro cultures.

The results obtained encourage the continuation of the biotechnological research related to the influence of different in vitro culture conditions on the biosynthetic ability of the investigated $H$. perforatum cultivars.

Open Access This article is distributed under the terms of the Creative Commons Attribution 4.0 International License (http://creati vecommons.org/licenses/by/4.0/), which permits unrestricted use, distribution, and reproduction in any medium, provided you give appropriate credit to the original author(s) and the source, provide a link to the Creative Commons license, and indicate if changes were made.

\section{References}

Barnes J, Anderson LA, Phillipson D (2007) St John's Worth. In: Herbal medicines. Pharmaceutical Press, London, pp 549-569

Beerhues L (2011) Biosynthesis of the active Hypericum perforatum constituents. In: Odabas MS, Çırak C (eds) Hypericum. Med Arom Plant Sci Biotechnol 5:70-77

Charchoglyan A, Abrahamyan A, Fujii I, Boubakir Z (2007) Differential accumulation of hyperforin and secohyperforin in Hypericum perforatum tissue cultures. Phytochemistry 68:2670-2677

Cirak C, Radusiěnë J, Karabük BS, Janulis V, Ivanauskas L (2007) Variation of bioactive compounds in Hypericum perforatum growing in Turkey during its phenological cycle. J Integr Plant Biol 49:615-620
Cui XH, Chakrabarty D, Lee EJ, Paek KY (2010) Production of adventitious roots and secondary metabolites by Hypericum perforatum L. in a bioreactor. Bioresour Technol 101:4708-4716

Dias ACP, Seabra RM, Andrade PB, Fernandes-Ferreira M (1999) The development and evaluation of an HPLC-DAD method for the analysis of the phenolic fractions from in vivo and in vitro biomass of Hypericum species. J Liq Chromatogr Relat Technol 22:215-227

Don Palmer C, Keller WA (2011) Plant regeneration from petal explants of Hypericum perforatum L. Plant Cell Tiss Organ Cult 105:129-134

Ekiert H (2004) Accumulation of biologically active furanocoumarins within in vitro cultures of medicinal plants. In: Ramawat KG (ed) Biotechnology of medicinal plants. Vitalizer and therapeutic. Science Publishers, Enfield, pp 267-296

Ekiert H, Szewczyk A, Kuś A (2009) Free phenolic acids in Ruta graveolens $\mathrm{L}$. in vitro culture. Pharmazie 64:694-696

Ekiert H, Kwiecień I, Szopa A (2013) Rosmarinic acid production in plant in vitro cultures. Pol J Cosmet 16:49-58

Ekiert H, Piekoszewska A, Muszyńska B, Baczyńska S (2014) Accumulation of $p$-cumaric acid and other bioactive phenolic acids in in vitro culture of Ruta graveolens ssp. divaricata (Tenore) Gams. Med Int Rev 26:24-31

Ellnain-Wojtaszek M, Zgórka G (1999) High-performance liquid chromatography and thin-layer chromatography of phenolic acids from Ginkgo biloba $L$. leaves collected within vegetative period. J Liq Chromatogr Relat Technol 22:1457-1471

Goel MK, Kukreja AK, Bisht NS (2009) In vitro manipulations in St. John's wort (Hypericum perforatum L.) for incessant and scale up micropropagation using adventitious roots in liquid medium and assessment of clonal fidelity using RAPD analysis. Plant Cell Tiss Organ Cult 96:1-9

Jurgenliemk G, Nahrstedt A (2002) Phenolic compounds from Hypericum perforatum. Planta Med 68:88-91

Kampa M, Alexaki VI, Notas G, Nifli AP, Nistikaki A, Hatzoglou A, Bakogeorgou E, Kouimtzoglou E, Blekas G, Boskou D, Gravanis A, Castanas E (2004) Antiproliferative and apoptotic effects of selective phenolic acids on T47D human breast cancer cells: potential mechanisms of action. Breast Cancer Res 6:63-74

Karting T, Gobel I, Heydel B (1996) Production of hypericin, pseudohypericin and flavonoids in cell cultures of various Hypericum species and their chemotypes. Planta Med 62:51-53

Khadem S, Marles R (2010) Monocyclic phenolic acids; hydroxyand polyhydroxybenzoic acids: occurrence and recent bioactivity studies. Molecules 15:7985-8005

Linsmaier EM, Skoog F (1965) Organic growth factor requirements of tobacco tissue cultures. Physiol Plant 18:100-127

Liu XN, Zhang XQ, Zhang SX, Sun JS (2007) Regulation of metabolite production by precursors and elicitors in liquid cultures of Hypericum perforatum. Plant Cell Tiss Organ Cult $91: 1-7$

Makri O, Kintzios S (2004) In vitro rosmarinic acid production: an update. In: Ramawat KG (ed) Biotechnology of medicinal plants. Vitalizer and therapeutic. Science Publishers, Enfield, pp 19-31

Murashige T, Skoog F (1962) A revised medium for rapid growth and bioassays with tobacco tissue cultures. Physiol Plant 15:473-497

Murch SJ, Saxena PK (2006) St. John's wort (Hypericum perforatum L.); challenges and strategies for production of chemically consistent plant. Can J Plant Sci 86:765-771

Muszyńska B, Ekiert H, Kwiecień I, Maślanka A, Zodi R, Beerhues L (2014) Comparative analysis of therapeutically important indole compounds in in vitro cultures of Hypericum perforatum 
cultivars by HPLC and TLC analysis coupled with densitometric detection. Nat Prod Commun 9:1437-1440

Pasqua G, Agato P, Monacelli P, Santamaria AR, Argentieri AP (2003) Metabolites in cell suspension cultures, calli, and in vitro regenerated organs of Hypericum perforatum cv. Topas. Plant Sci 165:977-982

Rice-Evans CA, Miller NJ, Paganga G (1996) Structure-antioxidant activity relationships of flavonoids and phenolic acids. Free Radic Biol Med 20:933-956

Sánchez-Maldonado AF, Schieber A, Gänzle MG (2011) Structurefunction relationships of the antibacterial activity of phenolic acids and their metabolism by lactic acid bacteria. J Appl Microbiol 111:1176-1184

Savio LEB, Astarita LV, Santarem ER (2012) Secondary metabolism in micropropagated Hypericum perforatum L. grown in non-aerated liquid medium. Plant Cell Tiss Organ Cult 108:465-472

Silva BA, Malva JO, Dias A (2008) St. John's Wort (Hypericum perforatum) extracts and isolated phenolic compounds are effective antioxidants in several in vitro models of oxidative stress. Food Chem 110:611-619

Szopa A, Ekiert H (2011) Lignans in Schisandra chinensis in vitro cultures. Pharmazie 66:633-634
Szopa A, Ekiert H (2012) In vitro cultures of Schisandra chinensis (Turcz.) Baill. (Chinense Magnolia Vine) - a potential biotechnological rich source of therapeutically important phenolic acids. Appl Biochem Biotechnol 166:1941-1948

Szopa A, Ekiert H (2013) Production of deoxyschizandrin and $\gamma$ schizandrin in shoot-differentiating and undifferentiating callus cultures of Schisandra chinensis (Turcz.) Baill. (Chinese magnolia vine). J Biotechnol 165:209-213

Szopa A, Ekiert H (2014) Production of biologically active phenolic acids in Aronia melanocarpa (Michx.) Elliott in vitro cultures cultivated on different variants of the Murashige and Skoog medium. Plant Growth Regul 72:51-58

Szopa A, Ekiert H (2015) Production of schisantherin A and gomisin $\mathrm{G}$ in in vitro cultures of Schisandra chinensis. Phytochem Lett 11:440-444

Szopa A, Ekiert H, Muszyńska B (2013) Accumulation of hydroxybenzoic acids and other biologically active phenolic acids in shoot and callus cultures of Aronia melanocarpa (Michx.) Elliot (black chokeberry). Plant Cell Tiss Organ Cult 113:323-329

Tatsis EC, Boeren S, Exarchou V, Troganis AN, Vervoort J, Gerothanassis IP (2007) Identification of the major constituents of Hypericum perforatum by LC/SPE/NMR and/or LC/MS. Phytochemistry 68:383-393 